\title{
Encefalomielitis aguda diseminada de presentación atípica: utilidad de la biopsia estereotáctica para su diagnóstico. Caso clínico
}

\author{
Pedro Vázquez $\mathbf{S}^{1}$, Andrés Reccius $\mathbf{M}^{1}$, Mario Díaz $\mathbf{S}^{1}$, \\ Lía Sáez $U^{2}$, Patricia 0 rellana $P^{3}$, Cristián Valdés $W^{1}$, \\ Violeta Díaz T¹. \\ Diagnosis of acute disseminated \\ encephalomyelitis using a stereotaxic \\ biopsy. Report of one case
}

We report a 15 year-old female presenting with behavioral disturbances, headache, left hemiparesis and paresis of the vertical gaze. CAT scan and magnetic resonance showed an involvement of right thalamus, third ventricle and medial temporal lobe suggesting an encephalitis or lymphoma. ${ }^{201}$ Thalium SPECT suggested a lymphoma. A stereotaxic biopsy showed a subacute demyelinizing lesion, compatible with an acute disseminated encephalomyelitis. The patient was treated with Methylprednisolone with resolution of symptoms. She remains in good condition after one year of follow-up (Rev Méd Chile 2006; 134: 883-6).

(Key w ords: Biopsy, needle; encephalomyelitis; Methylprednisolone)

Recibido el 15 de septiembre, 2005. Aceptado el 29 de noviembre, 2005.

Departamentos de ${ }^{1}$ Neurología-Neurocirugía, ${ }^{2}$ Anatomía Patológica e ${ }^{3}$ Imagenología, Hospital Clínico de la Universidad de Chile. Santiago de Chile.

L a encefalomielitis aguda diseminada (ADEM) es una enfermedad inflamatoria desmielinizante del sistema nervioso central (SNC). Se caracteriza por presentar defectos neurológicos multifocales, de inicio agudo o subagudo, habitualmente de curso monofásico, y por lo general presenta una buena recuperación. Puede ser precedida por una infección viral o una vacunación ${ }^{1}$. La ADEM afecta principalmente a niños y adultos jóvenes. Posiblemente, una exposición a un antígeno (infección o vacuna) gatillaría una respuesta inmune mediada

Correspondencia a: Dr. Pedro Vázquez. Neurocirugía. Santos Dumont 999. Teléfono: 7773882. Fax: 7378546. E mail: pvazquezs@yahoo.es por células $\mathrm{T}$ contra la proteína básica de la mielina u otro autoantígeno. Esto estaría causado por un mimetismo molecular o por activación inespecífica de un clon de células $\mathrm{T}$ autorreactivas ${ }^{2}$.

El proceso diagnóstico puede llegar a ser muy complejo. Se basa en el cuadro clínico, los hallazgos en la resonancia nuclear magnética cerebral (RNM) y el líquido cefalorraquídeo, debiéndose realizar, ocasionalmente, un estudio histopatológico, con biopsia de la lesión del cerebro $^{3-5}$. Según su forma de presentación y las neuroimágenes se plantea el diagnóstico diferencial con esclerosis múltiple, otras enfermedades desmielinizantes, encefalitis y procesos expansivos del $\mathrm{SNC}^{4,5}$. El objetivo de la presente publicación es mostrar un caso clínico, en donde la 
biopsia estereotáctica fue fundamental en el diagnóstico y su posterior enfrentamiento terapéutico.

\section{CASO CĹNICO}

Paciente de sexo femenino, de 15 años de edad, sin antecedentes mórbidos de importancia, sin cuadros infecciosos o vacunaciones en los meses previos. Ingresó al Hospital Clínico de la Universidad de Chile por presentar un cuadro de 3 semanas de evolución, caracterizado por cambios conductuales, puerilidad y desinhibición. Se agregó un síndrome confusional y disminución de la fuerza en el hemicuerpo izquierdo, asociado a cefalea holocránea leve a moderada. En el examen neurológi$\mathrm{co}$, al ingreso, destacaba una paciente vigil, pero confusa, con pupilas simétricas fotorreactivas, fondo de ojo sin papiledema, parálisis del $6^{\circ} \mathrm{par}$ craneal derecho, limitación en la mirada vertical ascendente, hemiparesia izquierda leve, y reflejo cutáneo-plantar extensor (Babinski) a izquierda.

La tomografía computada (TC) mostró una moderada hipodensidad alrededor del cuerno tem- poral derecho y otra que comprometió el tálamo del mismo lado, con escaso efecto de masa (Figura 1a) y sin captación significativa de medio de contraste (Figura 1b). En la resonancia nuclear magnética (RNM) se observaron en la secuencia FLAIR extensas lesiones hiperintensas a nivel temporal mesial derecho medial al cuerno temporal (Figura 1c), a nivel del tálamo del mismo lado y alrededor del tercer ventrículo (Figura 1d). También se observó un pequeño foco hiperintenso en la región subinsular anterior izquierda. Estas lesiones presentaban escaso efecto de masa y una evidente alteración de la barrera hematoencefálica, objetivado por un importante incremento de la señal T1, después del uso de Gadolinio, en la periferia de las lesiones, especialmente en aquella adyacente al cuerno temporal (Figuras 1e y 1f). Los hallazgos descritos plantearon los diagnósticos posibles de encefalitis o linfoma, $\mathrm{y}$, menos probable, una lesión desmielinizante pseudotumoral.

Los exámenes generales estaban dentro de límites normales. El estudio inicial de líquido cefalorraquídeo (LCR) mostró 20 leucocitos (100\% mononucleares) con glucosa y proteínas normales.

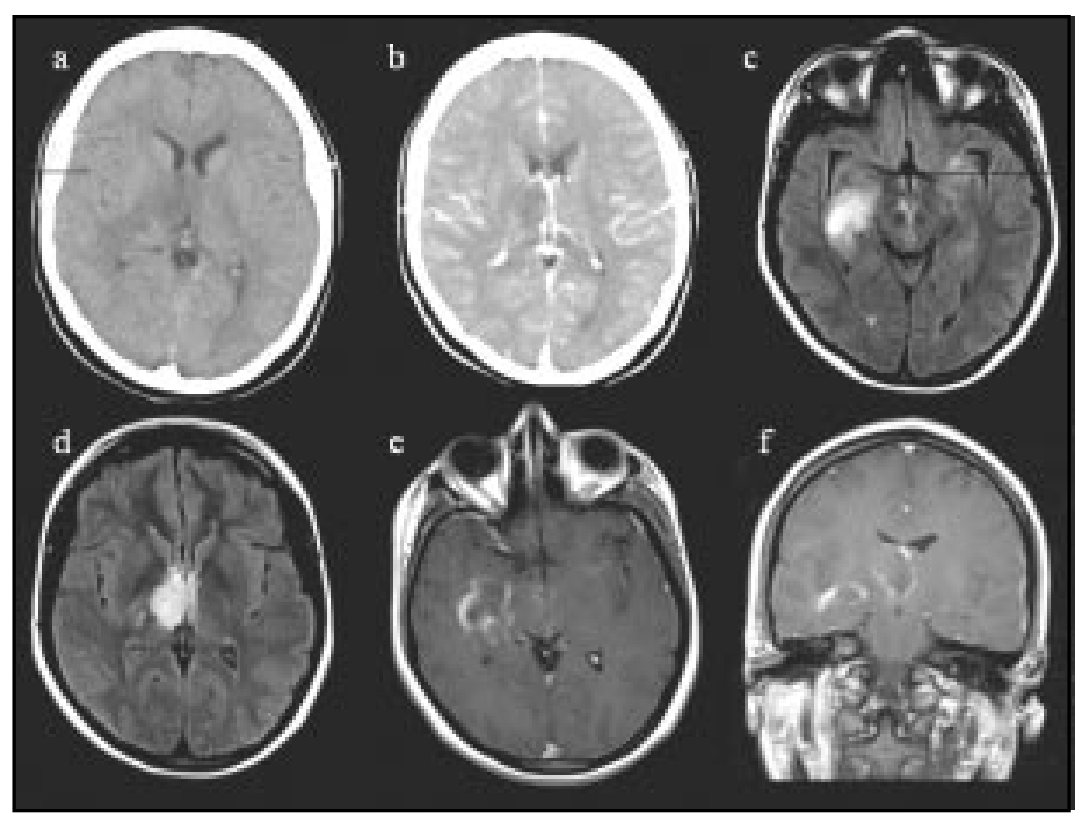

Figura 1. a) y b) TC Cerebral (a) sin medio de contraste, que muestra moderada hipodensidad talámica derecha y (b) con medio de contraste con escasa captación del mismo. c) y d) Secuencias FLAIR de RNM de Cerebro, (c) revela lesiones hiperintensas a nivel temporal mesial, (d) a nivel talámico derecho y alrededor del tercer ventrículo. e) y f) Secuencias T1 de RNM de cerebro con gadolinio de RNM (axial y coronal, respectivamente), muestran importante captación del medio paramagnético en relación a las lesiones descritas. 
Con estos elementos clínicos y ante la gravedad de una posible encefalitis herpética, se inició tratamiento empírico con aciclovir.

Los estudios inmunológicos (FR, ANA, ENA, ANCA) y de linfoma sistémico (LDH, ß2 microglobulina, TC de tórax y abdomen) fueron negativos. La PCR para virus herpes, el estudio de células neoplásicas, las bandas oligoclonales y el índice albúmina/IgG en el LCR resultaron normales. Se suspendió el tratamiento antiviral y se inició tratamiento con betametasona (4 mg cada $8 \mathrm{~h}$ ) para disminuir el edema cerebral, ante la posibilidad de un proceso expansivo.

Sin llegar a un diagnóstico aún, se realizó un SPECT cerebral con Talio 201, que mostró un foco de leve captación en el tálamo derecho que se extendía hacia temporal, sugerente de linfoma, más que lesión desmielinizante (Figura 2a).

La paciente evolucionó con deterioro neurológico progresivo, caracterizado por mayor compromiso de conciencia, llegando al sopor medio, compromiso total de la mirada vertical y hemiplejia izquierda.

Dada la gravedad de la paciente y la falta de diagnóstico, se decidió realizar una biopsia este-

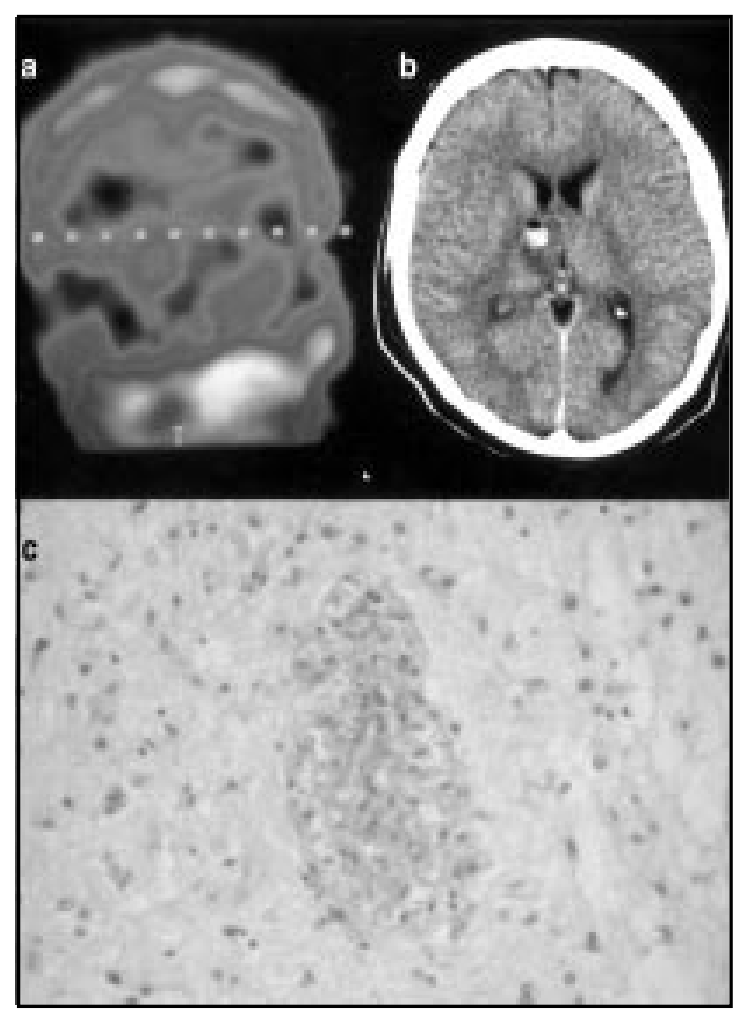

reotáctica. Se utilizó un anillo de arco centrado y un sistema de planificación computacional tridimensional, obteniendo así las coordenadas y ángulos precisos para llegar al blanco, sin lesionar tejido cerebral elocuente, a través de un abordaje precoronal derecho. Se tomaron muestras del centro de la lesión talámica, procedimiento sin complicaciones (Figura $2 b$ ).

El estudio histológico de la biopsia mostró un infiltrado inflamatorio discreto, de predominio mononuclear, de distribución preferentemente perivascular y gliosis reactiva, constituida por gemistocitos y macrófagos de citoplasma espumoso con granulaciones PAS (+). Estos hallazgos son compatibles con una patología desmielinizante de curso subagudo (Figura 2c).

Se inició tratamiento con metilprednisolona en dosis altas (un gramo al día por seis días), con lo que la paciente presentó una mejoría notoria, tanto de su compromiso de conciencia, como de sus defectos neurológicos focales. No volvió a presentar nuevos episodios transcurrido un año de evolución.

\section{Discusión}

En esta paciente el diagnóstico histológico fue compatible con una lesión desmielinizante subaguda. Creemos que por la historia clínica, las neuroimágenes y el estudio del LCR (ausencia de bandas oligoclonales y normalidad del índice albúmina Ig/

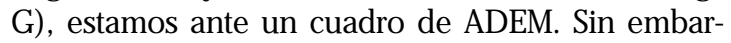
go, no se puede descartar un primer brote de una esclerosis múltiple (EM).

Figura 2. a) SPECT cerebral con Talio ${ }^{201}$. Foco de leve captación talámico derecho que se extiende hacia temporal. b) TC cerebral post biopsia, muestra burbuja de aire en el tálamo derecho en relación al sitio de biopsia. c) Corte histológico con tinción PAS a 1200 x. Infiltrado inflamatorio mononuclear perivascular y gliosis reactiva (gemistocitos y macrófagos con granulaciones PAS +). 
Algunos estudios de RNM han demostrado elementos que son más caractenísticos de ADEM que de EM, como la distribución de las lesiones en sustancia blanca subcortical más que periventricular, y el compromiso de la sustancia gris, tanto cortical como profundo, particularmente de los tálamos ${ }^{4,5}$. Además, se ha encontrado que las lesiones desmielinizantes de la ADEM tienden a ser pobremente marginadas ${ }^{6}$. Finalmente, lo más importante en su diferenciación es que en aquellos pacientes con ADEM de curso monofásico, se ha encontrado en los estudios imagenológicos de control una resolución de las lesiones o gliosis residual, y ausencia de nuevas lesiones, a diferencia de la esclerosis múltiple donde habitualmente aparecen nuevas lesiones asintomáticas?

El diagnóstico de la ADEM puede llegar a ser muy difícil, por lo que ocasionalmente se requiere realizar una biopsia cerebral, para demostrar una lesión desmielinizante. Schwarz ${ }^{8}$, en una serie retrospectiva de 40 casos de ADEM, recurrió a la biopsia en cuatro casos; Tenembaum ${ }^{10}$, en una

\section{REFERENCIA}

1. Bennetto L, Scolding N. Inflammatory/post-infectious encephalomyelitis. J Neurol Neurosurg Psychiatry 2004; 1: 22-8.

2. GARG RK. Acute Disseminated Encephalomyelitis. Postgrad Med J 2003; 79: 11-7.

3. Pohl-Koppe A, Burchett SK, Thiele EA, Hafier DA. Myelin basic protein reactive Th2 $\mathrm{T}$ cells are found in acute disseminated encephalomyelitis. J Neuroimmunol 1998; 91: 19-27.

4. Caldemeyer KS, Smith RR, Harris TM, Edwards MK. MRI in acute disseminated encephalomyelitis. Neuroradiology 1994; 36: 216-20.

5. Singh S, Alexander M, Korah IP. Acute disseminated encephalomyelitis: MR imaging futures. AJR Am J Roentgenol 1999; 173: 1101-7.

6. Dale RC, De Sousa C, Chong WK, Cox TC. Acute disseminated encephalomyelitis, multiphasic disseminated encephalomyelitis and multiple sclerosis in children. Brain 2000; 123: 2407-22.

7. HaRtung HP, GRossman R. ADEM distinct disease or part of the MS spectrum? Neurology 2001; 56: 1257-60.

8. Schwarz S, Mohr A, Knauth M, Wildemann B, Storch-Hagenlocher B. Acute disseminated encephalomyelitis: a follow-up study of 40 serie pediátrica de 84 pacientes, la utilizó en dos casos; Leake ${ }^{11}$, en uno de 42 casos y Hynson ${ }^{12}$ en dos de 31 pacientes. Murthy ${ }^{9}$, en otra serie pediátrica de 18 pacientes, no describe el uso de la biopsia.

En esta paciente, el diagnóstico previo a la biopsia no fue concluyente. La RNM planteaba una probable encefalitis o un proceso expansivo, el estudio de LCR no apoyó ninguna de estas dos etiologías y el SPECT fue compatible más bien con una lesión tumoral. La paciente presentó un rápido deterioro clínico, por lo que frente a un diagnóstico incierto, se recumó a la biopsia estereotáctica, que mostró finalmente una lesión desmielinizante, por lo que se pudo iniciar tratamiento con excelente respuesta.

Se destaca la utilidad de la biopsia estereotáctica en el diagnóstico de lesiones cerebrales de características inciertas. Es una técnica segura, con baja tasa de complicaciones, que van de 1 a $3 \%{ }^{13-}$ 16. En este caso en particular, la biopsia estereotáctica nos ayudó a definir la etiología, con lo que se pudo realizar un manejo adecuado y oportuno.

adult patients. Neurology 2001; 56: 1313-8.

9. Murthy SN, Faden HS, Cohen ME, BaKshi R. Acute disseminated encephalomyelitis in children. $\mathrm{Pe}$ diatrics 2002; 110: 1-7.

10. Tenembaum S, Chamoles $\mathrm{N}$, Fejerman N. Acute disseminated encephalomyelitis: a long-term follow-up study of 84 pediatric patients. Neurology 2002; 59: 1224-31.

11. Leake JA, Aiban S, Kao AS, Senac MO, Bшiman GF, NESPECA MP ET AL Acute disseminated encephalomyelitis in childhood: epidemiologic, clinical and laboratory features. Pediatr Infect Dis J 2004; 23: 756-64.

12. Hynson JL, Kornberg AJ, Coleman LT, Shield L, HaRvey AS, KeAn MJ. Clinical and neuroradiologic features of acute disseminated encephalomyelitis in children. Neurology 2001; 56: 1308-12.

13. V́́zquez P. Biopsia esterotáctica guiada por TAC y asistida por ordenador. Rev Chil Neurocirug 2004; 22: 76-82.

14. Bernstein M, PARRENT AG. Complications of CTguided stereotactic biopsy of intra-axial brain lesions. J Neurosurg 1994; 81: 165-8.

15. ApuZzo ML, SABshin JK. Computed tomographic guidance stereotaxis in the management of intracranial mass lesions. Neurosurgery 1983; 12: 277-85.

16. KeLY PJ. Stereotactic surgery: what is past is prologue. Neurosurgery 2000; 46: 16-27. 